\title{
Design and Development of Experimental Teaching System for Electric Drives Control System Course
}

\author{
Lv Hongli
}

Department of Information Engineering, Tangshan College, Tangshan City, Hebei Province, China (LHL741127@163.com)

\begin{abstract}
The paper is designed and developed the experimental teaching system for the course of electric drives control system according to the theoretical and practical characters of course. The simulation result is obtained by MATLAB/Simulink software platform and man-machine interface is designed by GUI to set parameters and input process data. The operation of teaching system is simple and intuitive. The simulation results are in accord with curriculum and it can be help students for deep understanding of the course.
\end{abstract}

Keywords - experimental teaching system, electric drives control system, man-machine interface, simulation

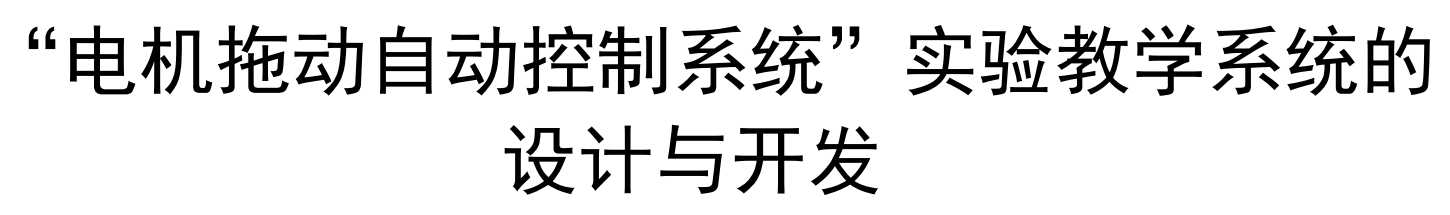

吕宏丽

唐山学院信息工程系，唐山，河北，中国

摘 要 本文针对 “电机拖动自动控制系统” 本科课程理论性和实践性较强的特点, 设计并开发了实验教学系统。该系统利用 MATLAB/Simulink 平台获得各实验的仿真结果, 通过 GUI 设计人机界面实现各种参数的修改, 整个实验教学系统操作简单直观, 仿 真结果与课程内容一致, 能够有效协助学生对课程内容的深入理解。

关键词＼cjkstart实验教学系统, 电机控制系统, 人机界面, 仿真

\section{1. 引言}

“电机拖动自动控制系统” 是高等院校电气工程及其 自动化本科专业的一门重要专业基础课程, 主要介绍直流 电机调速和交流电机调速的实现方法, 涉及到自动控制原 理、电力电子技术、计算机控制等多门课程知识, 概念多、 理论性强和工程实践联系密切 ${ }^{[1]}$ 。目前, 虽然有很多的实 验装置用于该课程的教学辅助, 但是由于系统被控对象 ——电机的动态过渡过程时间短、且含有很多非线性因素, 常规的仪表和示波器难以对其进行较好地测试与观察, 影 响了学生对该课程的理论学习。

MATLAB 是由美国 Mathworks 公司于 1984 年正式推 出的, 是 “矩阵实验室” (MATrix LABoratoy) 的缩写。它是 一个高度集成的软件系统, 集科学与工程计算、图像处理、 图形可视化、多媒体处理于一身, 形成了一个方便的, 界 面友好的用户环境。Simulink 是 MATLAB 的可视化动态仿
真环境, 由于支持线性、非线性、连续、离散、多变量和 混合式系统结构, Simulink 几乎可分析任何一种类型的真 实动态系统, 使复杂系统的输入和仿真变得相当简单。利 用 MATLAB 软件中的 GUI 功能设计的人机界面可提供建 立系统模型、选择仿真参数和数值算法、启动仿真程序对 该系统进行仿真、设置不同的输出方式来观察仿真结果等 功能 ${ }^{[2]}$ 。因此本实验教学系统是采用 MATLAB 语言中的 Simulink 动态仿真环境对交直流电动机调速实验进行仿 真, 并利用 MATLAB 强大的 GUI (图形用户交互界面设 计）功能设计了实验教学系统, 促使学生加深对交直流电 动机调速原理的理解。

\section{2. 实验教学系统的设计}

在进行实验教学系统平台设计时, 始终把自动控制系 统理论的基本思想和核心概念作为设计思想, 以提高电子 
电路分析设计能力和电气传动系统分析设计能力为目标。

“电机拖动自动控制系统” 实验教学系统分为直流调 速系统和交流调速系统两部分 ${ }^{\left[{ }^{[3]}\right.}$, 主界面如图 1 所示。直 流电机调速系统有五个实验项目, 界面如图 2 所示。交流 电机调速系统有四个实验项目, 界面如图 3 所示。通过点 击相应的仿真实验, 进入对应的参数设置和运行界面。

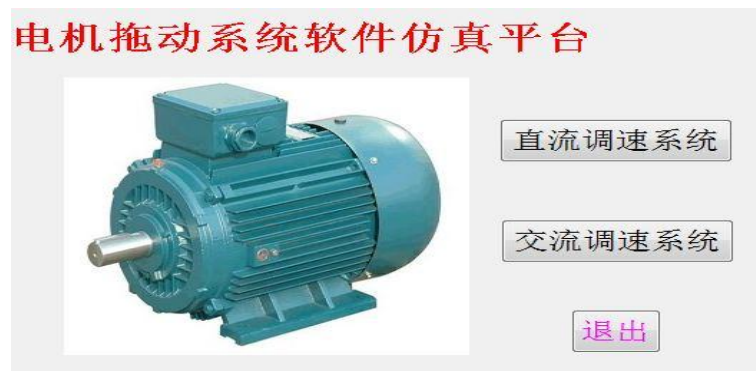

图 1 实验教学系统主界面

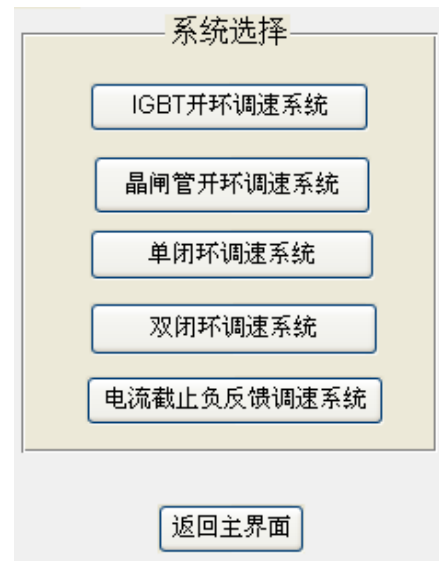

图2 直流电机调速系统实验项目界面

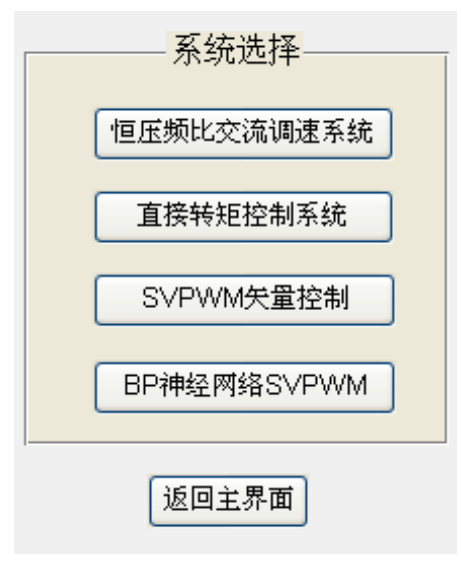

图3 交流电机调速系统实验项目界面

\section{3. 实验教学系统的具体实现}

以直流电机调速系统的双闭环调速系统实验为例。转 速、电流双闭环调速系统是比较经典的自动控制系统, 能 获得良好的静、动态性能, 控制器参数的设置方法是课程 的重点和难点内容。为了得到直观的控制效果, 图4为双闭 环直流调速系统的GUI界面。

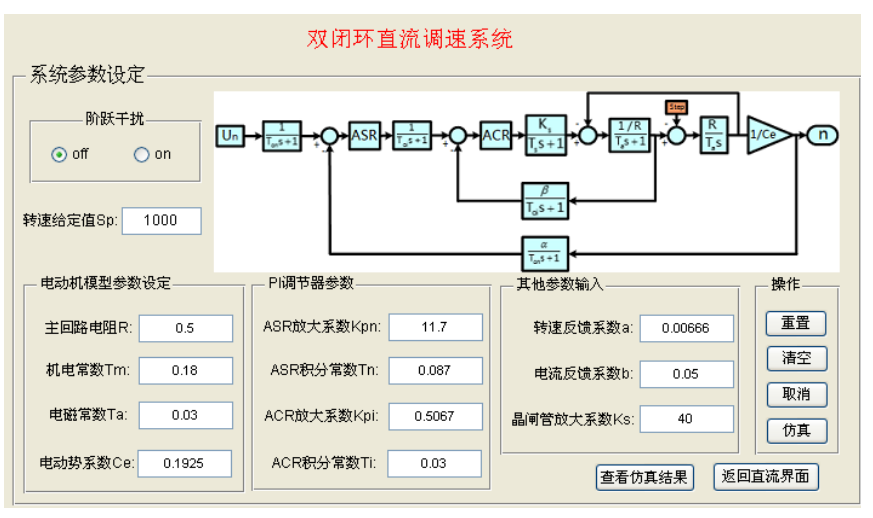

图4 转速电流双闭环直流调速系统的参数设置GUI界面

利用 imshow () 函数实现图 4 中控制系统原理图的显 示, 界面中需要设置电动机参数、调节器参数和其它参数, 使用 get () 函数获得文本框中的各种参数值, 利用 simset ( ) 函数将读取的数值写入 Simulink 模块中。功能按钮有 “重置”、“清空”、“取消”和“仿真”。“重置”按钮实现的功 能是将所有参数恢复初始状态赋给仿真模型; “清空”按钮 是将所有的模型参数都清空, 可由用户自定义模型参数; 点击“取消”按钮可退出仿真实验界面, 回到主界面; “仿真” 按钮的功能是运行后台的仿真模型文件。

通过图 4 界面设置好直流电动机模型参数、两个调节 器参数及其它参数, 阶跃干扰选项中点选 “off”, 点击“仿真” 按钮并“查看仿真结果” (如图 5), 观察到在没有负载扰动 情况下的转速电流波形, 同时显示各种动态性能指标值, 使双闭环控制系统的性能一目了然。该系统可以随意修改 调节器参数, 例如保持相同的 ASR (转速调节器) 参数, 将 ACR (电流调节器) PI 参数进行修改 (如图 6), 重新“仿 真”, 仿真波形如图 7 所示。对比图 5 和图 7 的波形曲线 和动态性能指标发现, 按图 7 设置参数的系统电枢电流反 应较快, 性能较好, 这与课程内容中讲解的参数变化趋势 一致。

学生使用本系统, 可通过任意修改转速调节器和电流 调节器的参数, 观察电压电流波形的改变, 帮助学生深入 理解调节器参数对系统性能的影响, 同时体会工程中利用 试凑法整定调节器参数的过程, 效果直观。 


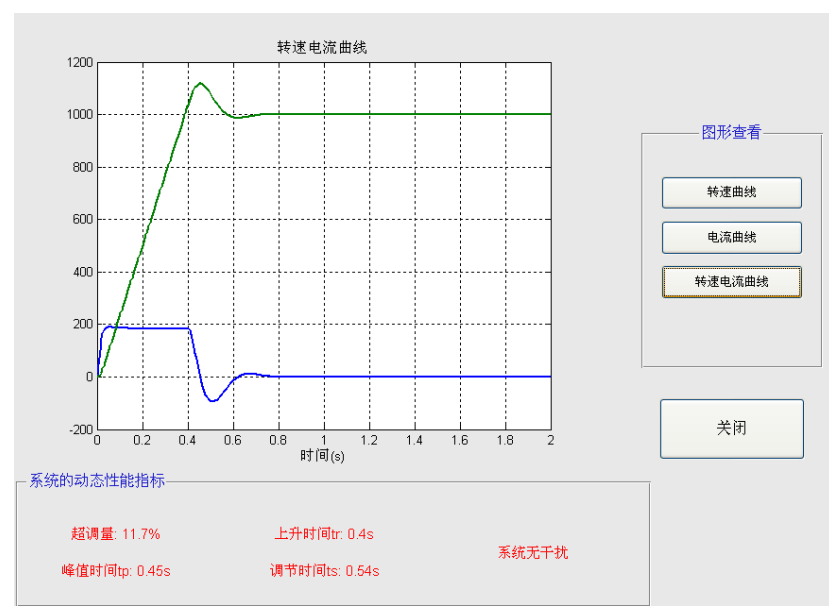

图 5 特定参数下无负载扰动时的转速电流仿真波形

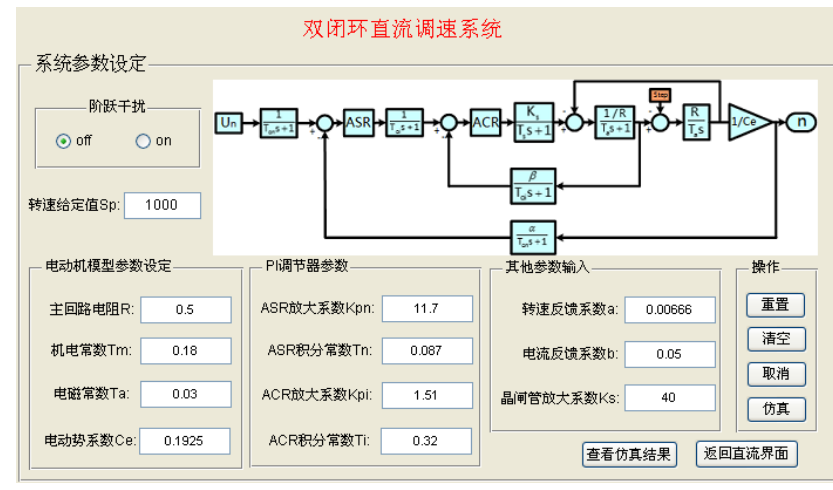

图 6 修改 $\mathrm{ACR}$ 参数

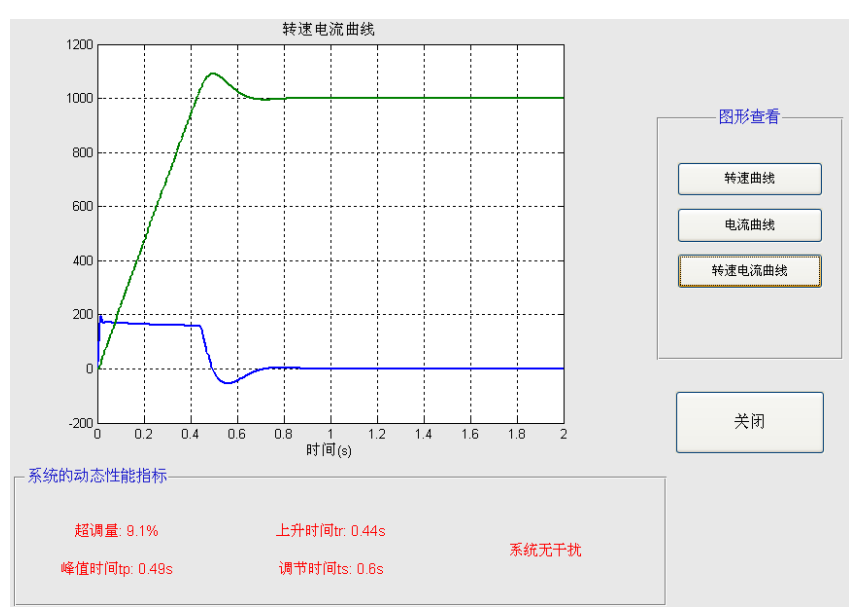

图 7 修改 $\mathrm{ACR}$ 参数后的转速电流仿真波形

当图 4 界面阶跃干扰选项中点选“on”, 将设置从空载 到满载的扰动, “仿真”后波形如图 8 所示, 不仅显示出跟 随动态性能指标, 而且显示出抗扰动态性能指标, 有助于 学生分析调节器参数对抗扰性能的影响程度。

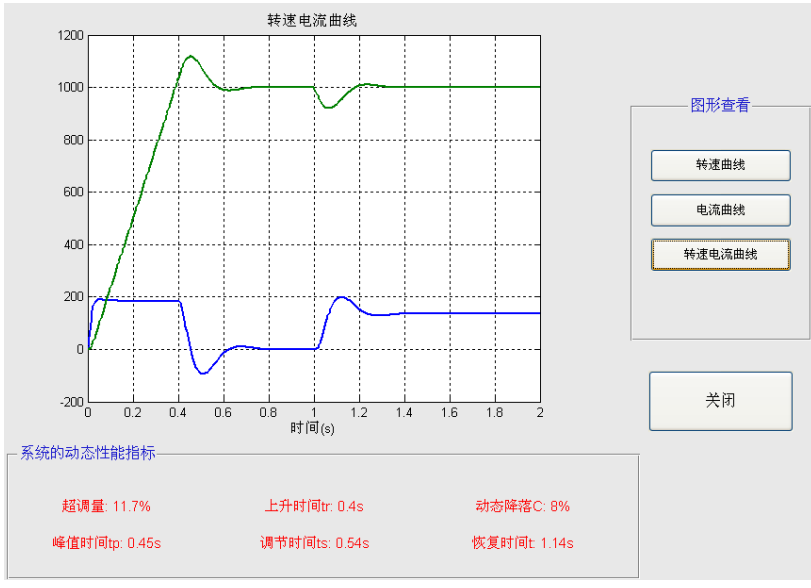

图 8 特定参数下有负载扰动时的转速电流仿真波形

交流电机调速部分以恒压频比交流调速系统实验为 例。恒压频比控制系统是基于交流异步电动机稳态模型, 采用稳态等值电路来分析异步电动机在不同电压和频率供 电条件下转矩与磁通的稳态关系和机械特性, 并在此基础 上设计异步电动机调速系统 ${ }^{[4]}$ 。由于异步电动机稳态模型 较为复杂, 理论性较强, 学生学习过程中多会把注意力集 中于稳态模型的推导中, 而忽略了研究稳态模型的意义。 通过实验教学系统的使用, 力图突出 “系统” 的概念, 将 学生的兴趣引导到控制系统是如何实现的, 系统性能指标 是如何达到的。图 9 为恒压频比交流调速系统实验的 GUI 界面。

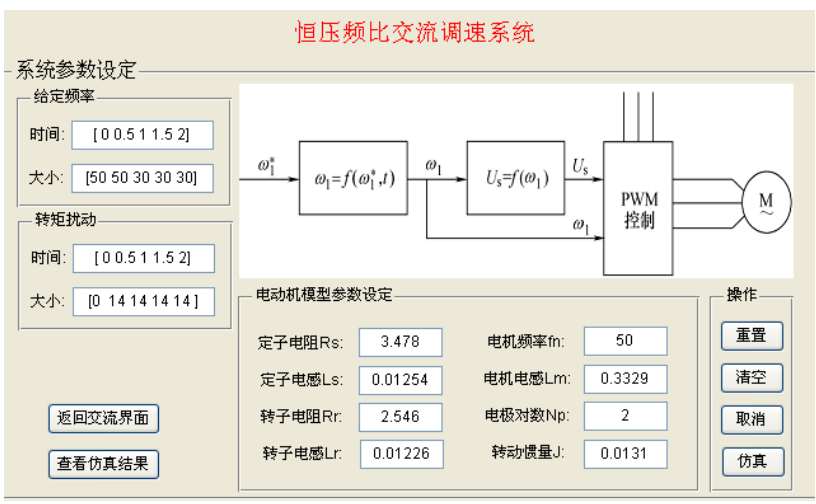

图 9 恒压频比控制系统的参数设置 GUI 界面

图 9 中给定频率和转矩扰动的设置采用矩阵形式, 便 于导入后台的仿真模型文件。按照图 9 参数的设置, 仿真 系统在 $0-0.5 \mathrm{~s}$ 时电动机空载, $0.5 \mathrm{~s}$ 后电机带额定负载, 同 时设置频率 $0-1 \mathrm{~s}$ 时为 $50 \mathrm{~Hz}, 1 \mathrm{~s}$ 之后降为 $30 \mathrm{~Hz}$ 。仿真结果 如图 10 所示, 可以显示三相电压、定子电流、转子电流、 电机转速、转矩、转矩扰动和 PWM 波形, 图 10 显示的是 电机转速的仿真曲线。通过各种仿真曲线的对比分析, 学 
生会直观的了解到在 $0-0.25 \mathrm{~s}$ 时间段内, 电机处于加速状 态, $0.25 \mathrm{~s}$ 后基本达到稳定, 由于电机是空载运行, 所以定 子电流为空载电流, 频率为 $50 \mathrm{~Hz}$, 转子电流基本为零, 转 矩也为零。在 $0.5 \mathrm{~s}$ 时, 负载突然变为满载, 定转子电流迅 速增加, 输出转矩跟随变化, 电机转矩振荡下降, 最终稳 定。当在 $1 \mathrm{~s}$ 时, 电源频率降为 $30 \mathrm{~Hz}$, 电机开始减速, 并 最终达到一个稳定值, 在此期间定子电流的频率减小。

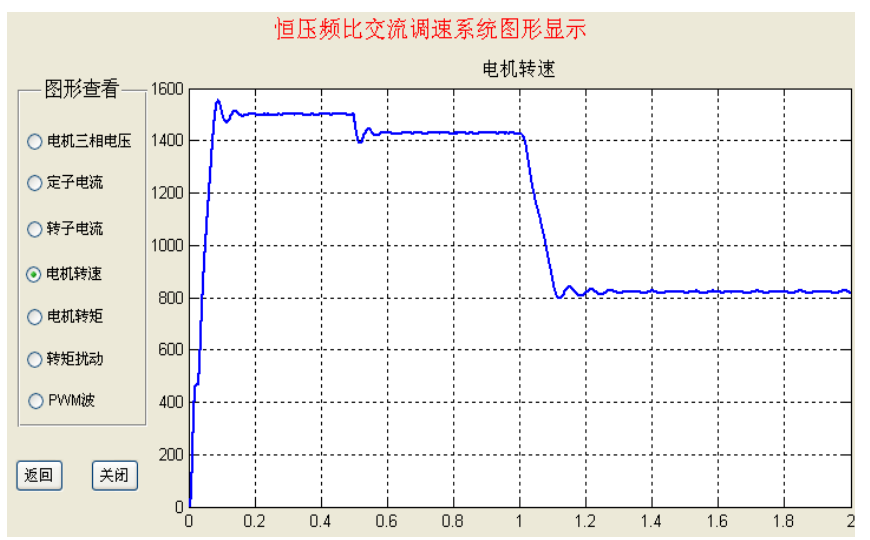

图 10 恒压频比交流调速系统的仿真曲线

当修改给定频率和转矩扰动的设置, 通过相应仿真曲 线的变化分析, 可对交流异步电动机各参数之间的关系有 深入理解, 这也是异步电动机稳态模型的实质。同时, PWM 控制是本实验的关键, 这部分是在 Simulink 中实现的。

为了拓宽学生视野, 在交流调速系统部分实验项目中 加入了神经网络控制内容, 将 BP 神经网络用于 SVPWM 控制实现交流调速 ${ }^{[5]}$, 使学习能力较强的学生对智能控制 在电机拖动控制系统中的应用有所了解, 为今后深入学习 打下基础。图 11 为 BP 神经网络 SVPWM 实验的 GUI 界面, 图 12 为定子磁链的仿真曲线。

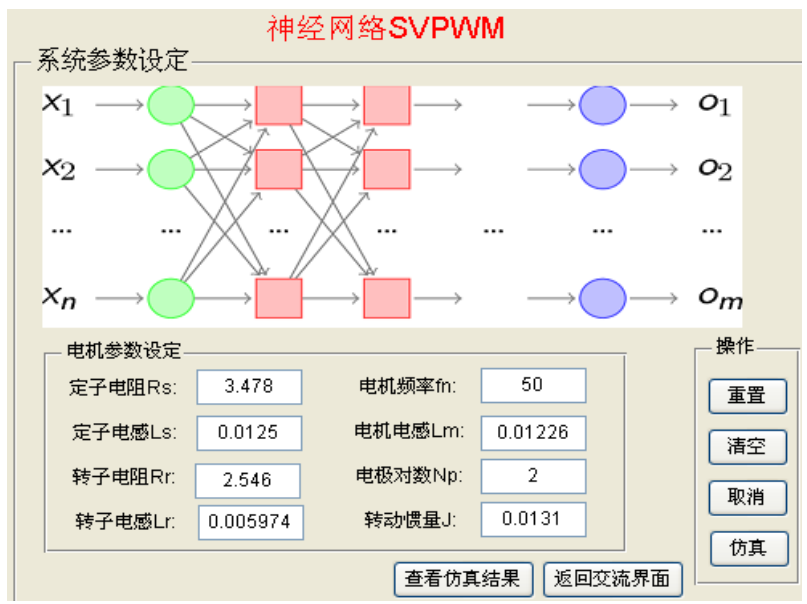

图 $11 \mathrm{BP}$ 神经网络 SVPWM 系统参数设置 GUI 界面

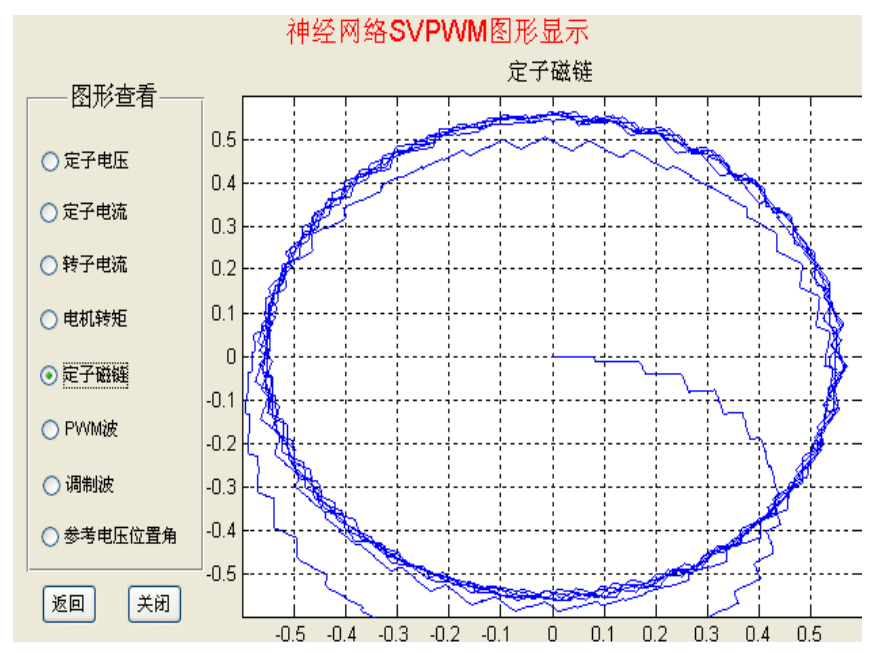

图 $12 \mathrm{BP}$ 神经网络系统定子磁链的仿真曲线

\section{4. 结论}

设计开发的实验教学系统具有可视化的特点, 操作方 便、直观。通过 GUI 界面, 操作人员可以进入任意的实验 项目, 链接 Simulink 仿真环境并打开实验项目的仿真模型, 设置和调整仿真参数, 进行仿真实验。通过仿真实验结果 进行比较分析, 有助于学生发现实验中存在的问题, 锻炼 独立思考问题、分析问题的能力。

\section{参考文献(References)}

[1] Ruan Yi, Chen Bo-shi. Control System of Electric Drives-Motion Control Systems. $4^{\text {th }}$ ed..Beijing:China Machine Press, 2009.8

[2] Wang Zheng-lin. Advanced Knowledge of MATLAB GUI. Beijing: Electronic Industry Press, 2008.2

[3] Shang Li, Huai Wen-jun. The Virtual Experiment Platform Design of Auto-control System Based on MATLAB/Simulink and GUI. Research and Exploration in Laboratory, 2010,(06)66-71

[4] Li Jun-biao. Research of PWM Frequency Control System Based on DSP. Inner Mongolia University of Science \& Technology, 2009.6

[5] Lv Hong-li, Ye Shuang. Simulation of Controlling System with SVPWM Based on BP Neural Network. Journal of Tangshan Teachers College, Vol.35 No.5, Sep.2013:49-51 